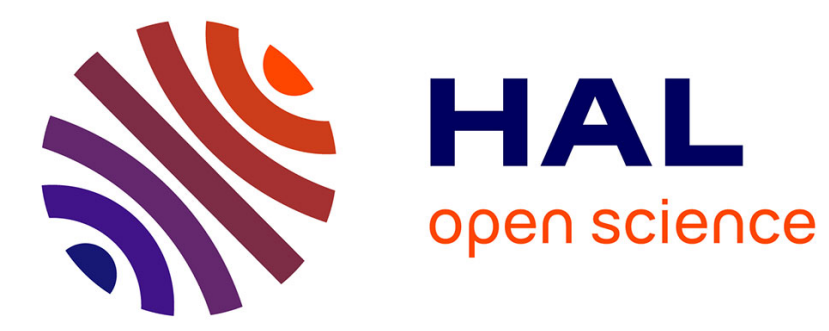

\title{
Small-scale intermittency in anisotropic turbulence
}

Wouter J.T. Bos, Liechtenstein Lukas, Kai Schneider

\section{To cite this version:}

Wouter J.T. Bos, Liechtenstein Lukas, Kai Schneider. Small-scale intermittency in anisotropic turbulence. Physical Review E: Statistical, Nonlinear, and Soft Matter Physics, 2007, 76, pp.046310. 10.1103/PhysRevE.76.046310 . hal-00557022

\section{HAL Id: hal-00557022 \\ https://hal.science/hal-00557022}

Submitted on 18 Jan 2011

HAL is a multi-disciplinary open access archive for the deposit and dissemination of scientific research documents, whether they are published or not. The documents may come from teaching and research institutions in France or abroad, or from public or private research centers.
L'archive ouverte pluridisciplinaire HAL, est destinée au dépôt et à la diffusion de documents scientifiques de niveau recherche, publiés ou non, émanant des établissements d'enseignement et de recherche français ou étrangers, des laboratoires publics ou privés. 


\title{
Small scale intermittency in anisotropic turbulence
}

\author{
Wouter J.T. Bos, Lukas Liechtenstein, Kai Schneider \\ MSNMGP - CNRS \& CMI - Université de Provence, Marseille, France
}

(Dated: September 17, 2007)

\begin{abstract}
Isotropic, rotating and stratified turbulent flows are analyzed using a scale- and directiondependent flatness. The anisotropy of the spatial fluctuations of the energy distribution can hereby be quantified for different length scales. This measure allows to distinguish between longitudinal and transversal intermittency as well as between horizontal and vertical intermittency. The difference between longitudinal and transversal intermittency is argued to be related to the incompressiblity constraint. A large difference between horizontal and vertical intermittency for stratified turbulence can be explained by an energy depletion of the horizontal plane in Fourier space.
\end{abstract}

PACS numbers: 47.27.-i, 47.27.Gs, 47.32.Ef

\section{INTRODUCTION}

Turbulence in geophysical flows, e.g. in the atmosphere or in the ocean, is generally anisotropic due to the presence of shear, rotation and stratification. In these flows the modeling and understanding of anomalous transport of pollutants needs knowledge about small scale intermittency. In the present work by intermittency we mean small scale activity, localized in space. Intermittency has been studied since its introduction by Taylor [1]. Townsend [2] was the first to introduce a measure for small scale intermittency, the flatness. Subsequently, to study the variation of intermittency with scale, Sandborn introduced a quantitative measure, the scale-dependent flatness [3], and showed that for intermittent flows the flatness strongly increases with the wavenumber.

Batchelor and Townsend [4] explained intermittency by the presence of coherent structures. As in turbulence the coherent structures reflect the anisotropy (e.g. vortex tubes aligned with the rotation axis in rotating turbulence and horizontal vortex sheets in stably stratified turbulence as shown in figure 1), the intermittency can be expected to be anisotropic. This issue, the anisotropy of small scale intermittency will be addressed in the present work.

To study anisotropic turbulence specific tools are needed. Progress has been made, notably by using directional energy spectra ([5-7] and references therein). Directional spectra, while capturing the multiscale character, can not quantify the spatially intermittent nature of the flows. Indeed the intermittency related to spatially localized events is hidden in the phase of the Fourier modes. It was argued by Brun and Pumir [8] that when the largest turbulent scales are well resolved, i.e. the domain-size is much larger than the correlation length, intermittency is only observable in the dissipation range. This disspation range intermittency was predicted by Kraichnan [9]: in the highest wavenumber range, where viscous dissipation smoothens the fluctuations, the distribution of energy shows a very fast fall-off. In this range, small variations of the cut-off scale, corresponding to the different flow structures or regions, lead to tremendous relative fluctuations of the turbulent intensity.
If, in addition to dissipation range intermittency, inertial range intermittency is of interest, Fourier modes are not the adequate basis functions. Wavelet coefficients are then more appropiate, because they conserve information on the localization in physical space. Another method is the use of structure functions, in particular within the context of the SO3 decomposition [10]. However, structure functions can yield erroneous scalings if the considered velocity field is too smooth or too irregular $[11,12]$. Indeed structure functions are closely related to wavelets [12], who do not have this disadvantage but conserve the other advantages of structure functions: they can simultaneously address the multiscale distribution of a quantity, its anisotropy and yield information on its spatial distribution. In the present work we therefore use wavelets and in particular a statistical tool is introduced, the scale-dependent directional flatness, inspired by the work of Meneveau [13] and of Farge et al. [14], who used three-dimensional orthogonal wavelets and two-dimensional angle-dependent continuous wavelets, respectively. The scale-dependent directional flatness can be seen as an anisotropic extension of the spectral flatness $[8,15,16]$, applied to scale space rather than to Fourier space.

In the next section we will describe the direct numerical simulations that yield the velocity fields analyzed in this work. Subsequently, in section 3 we will discuss the link between wavelet space and Fourier space. In section 4 we analyze the velocity fields using two measures: the directional energy and the scale-dependent directional flatness. It is shown that a large part of the observed anisotropy of the energy distribution is due to the incompressibility constraint. Finally, it is argued that the anisotropic small scale intermittency can be explained by the competition of various mechanisms: dissipation range fluctuations, incompressibility and body forces.

\section{DIRECT NUMERICAL SIMULATIONS}

We consider velocity fields obtained by direct numerical simulation (DNS) of Navier-Stokes turbulence, using a classical pseudo-spectral method. Anisotropy is created 
TABLE I: Sets of parameters of the three DNS runs. $\mathcal{T}_{0}$ indicates after how many turnover times the data is evaluated.

\begin{tabular}{l||c|c|c|c|c}
\hline & $e$ & $\mathcal{L}$ & $R_{\lambda}$ & $R o$ or $F r$ & time $\left(\mathcal{T}_{0}\right)$ \\
\hline Isotropic & 0.014 & 0.37 & 34 & & 6 \\
Rotating & 0.034 & 0.37 & 60 & $R o=0.025$ & 3 \\
Stratified & 0.056 & 0.44 & 121 & $F r=0.028$ & 11.5 \\
\hline
\end{tabular}

by rotation and stratification, both oriented along the vertical axis. The velocity fields considered here correspond to decaying isotropic, rotating and stably stratified incompressible turbulence within the Boussinesq approximation using $512^{3}$ grid points. No external forcing is applied to the velocity fields, so that the Reynolds number is moderate and the inertial range is not well pronounced. However, forcing is known to affect the anisotropy induced by the body forces. To avoid artefacts caused by the forcing, the freely decaying case is considered. The parameters are summarized in table I. Specific attention has been paid to the large scale anisotropy of the flow: the Froude and Rossby number are comparable. These quantities are defined as $F r=\mathcal{U} / \mathcal{L} N$ and $R o=\mathcal{U} / \mathcal{L} f$, with $N$ and $f$ being the Brunt-Väisälä frequency and rotation number, respectively. Here the integral turbulent velocity $\mathcal{U}$ and the integral lengthscale $\mathcal{L}$ are defined as

$$
\mathcal{U}=\sqrt{2 e / 3}, \quad \mathcal{L}=\frac{\pi}{2 \mathcal{U}^{2}} \sqrt{\int \frac{E(k)}{k} d k},
$$

with $e=\int E(k) d k$ and $E(k)$ the spherically averaged energy spectrum. The Reynolds numbers, based on the Taylor-microscale $\left(R_{\lambda}=\sqrt{20 e^{2} /(3 \nu \epsilon)}\right.$, with $\nu$ the kinematic viscosity and $\epsilon$ the dissipation of kinetic energy), are of the same order of magnitude. For details about the method and the set-up of the simulations we refer to [17]. In addition to these three cases a vector valued test field was generated consisting of divergence-free Gaussian white noise, with $E(k) \sim k^{2}$.

\section{WAVELET SPACE AND ITS RELATION TO FOURIER SPACE}

The velocity field at a given time instant is projected onto an orthogonal wavelet basis (see e.g. [18] for details). We use Coiflet-12 wavelets, which have 4 vanishing moments and a filterlength of 12 . The projection of one component $u(\boldsymbol{x})$ of a vector field $\boldsymbol{u}=(u, v, w)$, on orthogonal wavelets $\psi(\boldsymbol{x})$ can be represented by

$$
u(\boldsymbol{x})=\sum_{\lambda} \tilde{u}_{\lambda} \psi_{\lambda}(\boldsymbol{x})
$$

with the subscript $\lambda=(j, i, d)$, where $j$ represents the scale, $\boldsymbol{i}$ the position and $d$ the direction. The coefficients

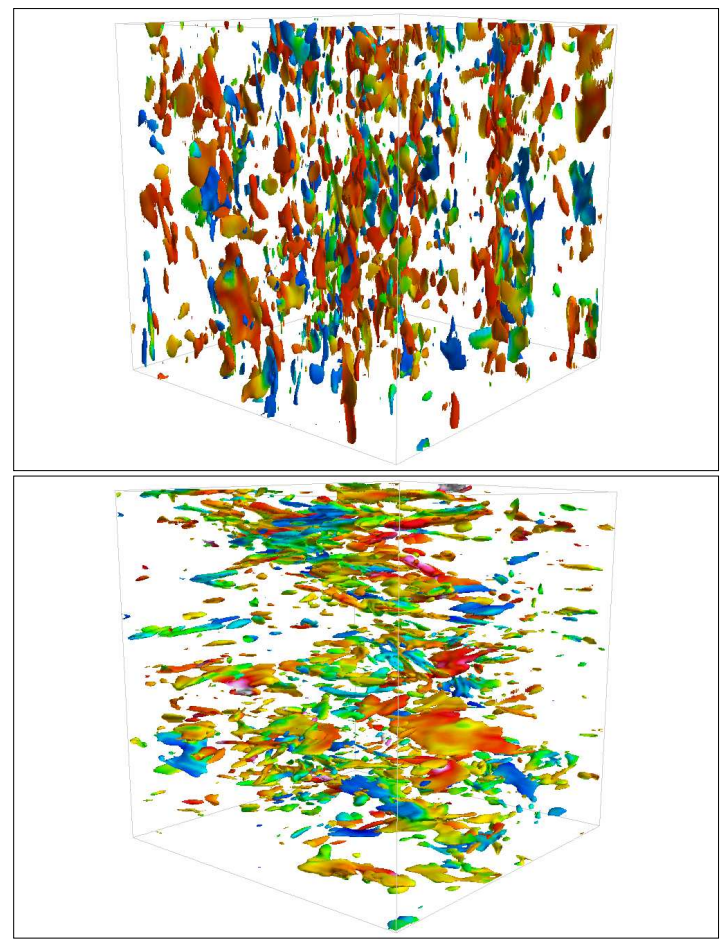

FIG. 1: (Color online) Iso-enstrophy surfaces from direct numerical simulation for rotating (top) and stratified (bottom) turbulence with an iso-value equal to the mean enstrophy. In the rotating case we observe elongated vertical structures, in the stratified case flattened horizontal structures. The vertical velocity is shown in the visualization by a color scale ranging from blue (negative velocity) to red (positive velocity).

are stocked in a $512^{3}$ wavelet space. The orthonormal character of the wavelets implies that for a datafield of $N^{3}=2^{3 J}$ values, $j$ takes the values $j=\{0,1, . ., J-1\}$. In the wavelet representation 7 spatial directions can be defined in three space dimensions (in $D$ dimensions, $2^{D}-1$ directions exist). For every particular combination of scale $j$ and direction $d, i$ can take $2^{3 j}$ different values, which give an information on the localization in physical space. The parameters $(j, i, d)$ are then equivalent to coordinates in wavelet space. This wavelet space, in the Mallat representation [19], is shown in figure 2 as compared to Fourier space for both two and three dimensions. Hereby a direct link is shown between the two approaches. The dashed part of wavelet space, corresponding to one of the 7 spatial directions (in three dimensions), is associated to the dashed part of Fourier space in the figures: it constitutes an orthogonal partition of Fourier space. Each individual box in wavelet space corresponds to a direction $d$ and a scale $j$. Each scale can be linked to a wavenumber $k_{j}$ by

$$
k_{j}=k_{0} 2^{j} \quad \text { with } \quad k_{0}=\frac{\int k|\hat{\psi}(k)| d k}{\int|\hat{\psi}(k)| d k}
$$

where $k_{0}$ is the centroid-wavenumber, a constant for each type of wavelet $\left(k_{0} \approx 0.77\right.$ for Coiflet-12). It is hereby 
possible to reconstruct an energy spectrum, by calculating the spectral energy density corresponding to a wavenumber $k_{j}$. The relation is

$$
\tilde{E}\left(k_{j}\right)=\alpha_{j}\left\langle e_{\lambda}\right\rangle_{(j)} \quad \text { with } \quad e_{\lambda}=\left(\tilde{u}_{\lambda}^{2}+\tilde{v}_{\lambda}^{2}+\tilde{w}_{\lambda}^{2}\right) / 2
$$

in which the averaging has been performed over the position $i$ and the direction $d$ and with $\alpha_{j}=4 \pi 2^{2 j} /\left(7 k_{0}\right)$, a scale-dependent factor relating the discrete wavelet representation to spherical shells in Fourier space. This approach was initially proposed by Meneveau [13] and for further details we refer to his pioneering work. The disadvantage of these wavelet based energy spectra, is the loss of spectral resolution: the spectrum contains one point for each scale $j$, that is, for each octave. This loss of information is however compensated by an information on the spatial variance of the energy spectrum, because for every scale $j$ (corresponding to 7 boxes), we have $2^{3 j} \times 7$ values. The spatial variation can be expressed as the standard deviation of the spectral distribution. This standard deviation is defined by:

$$
\sigma_{\tilde{E}}\left(k_{j}\right)=\alpha_{j}\left[\left\langle e_{\lambda}^{2}\right\rangle_{(j)}-\left\langle e_{\lambda}\right\rangle_{(j)}^{2}\right]^{1 / 2}
$$

In figure 3 both the spherically averaged Fourier energy spectrum $E(k)$ and the wavelet spectrum $\tilde{E}\left(k_{j}\right)$ are shown for isotropic turbulence. The wavelet spectrum agrees well with the Fourier spectrum. The spatial variation of the spectral distribution is shown in figure 3 by adding the points that indicate the spectrum plus one standard deviation $\left(\tilde{E}\left(k_{j}\right)+\sigma_{\tilde{E}}\left(k_{j}\right)\right)$. This variation is closely related to the flatness as will be explained in the following.

\section{ANALYSIS OF THE ANISOTROPY OF THE VELOCITY FIELDS}

\section{A. Directional energy}

Because of incompressibility, the energy distribution in Fourier space of an isotropic vector-field can be expressed as

$$
\Phi_{i j}(\boldsymbol{k})=\left(\delta_{i j}-\frac{k_{i} k_{j}}{k^{2}}\right) \frac{E(k)}{4 \pi k^{2}},
$$

which means that for a certain component (for example $\left.\Phi_{11}(\boldsymbol{k})\right)$ one finds:

$$
\Phi_{11}(\boldsymbol{k})=\left(1-\frac{k_{1} k_{1}}{k^{2}}\right) E(k)=\sin ^{2} \phi \frac{E(k)}{4 \pi k^{2}},
$$

with $\phi$ the angle between the wave vector and the $k_{x^{-}}$ axis. This results in an eight-shaped distibution in 2 dimensions, shown in figure 4 . In 3 dimensions this eight is rotated around the $k_{x}$-axis for $\Phi_{11}(\boldsymbol{k})$. This leads to an anisotropy in the directional energy of the vector field
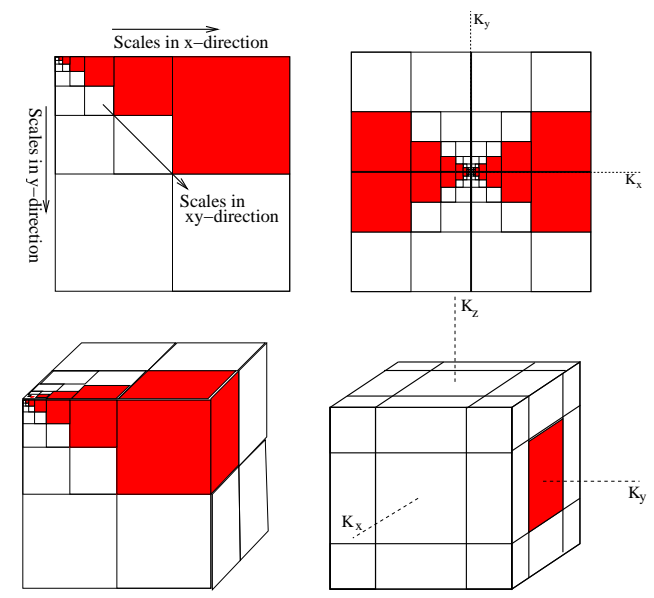

FIG. 2: (Color online) Left: wavelet space in two and three dimensions. Right: wavelet space projected onto Fourier space. Each box corresponds to a certain scale $j$ and direction $d$ and each box contains $2^{2 j}$ values of $\boldsymbol{i}$ in two dimensions and $2^{3 j}$ in three dimensions, giving information on localization in physical space. Hereby we can relate a scale $j$ to a wavenumber $k_{j}: \quad k_{j}=k_{0} 2^{j}$, where $k_{0}$ is the centroid-wavenumber of the wavelet.

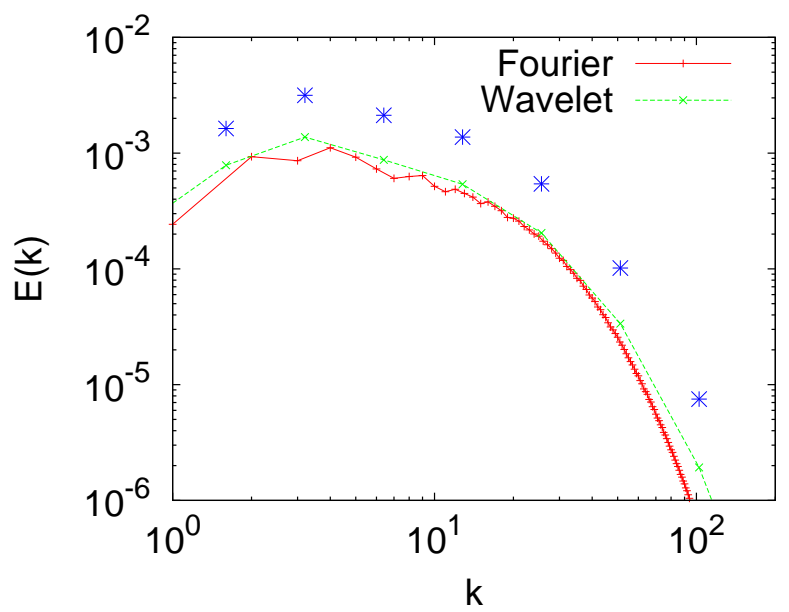

FIG. 3: (Color online) The (Fourier) energy spectrum for isotropic turbulence. Also shown is the dual spectrum constructed from wavelet coefficients. The stars indicate the standard deviation of the spatial distribution of the spectral energy density.

components, even for isotropic fields. Rotation or stratification can amplify or reduce this effect.

The general picture that emerges from studies using directional spectra $[6,20]$ is that in stratified turbulence the kinetic energy is concentrated in a cone around the vertical axis of Fourier space. For rotating turbulence, the inverse tendency, an accumulation of energy close to the horizontal plane in Fourier space, is observed. In physical space this energy distribution corresponds to sheet-like structures in stratified turbulence and verti- 


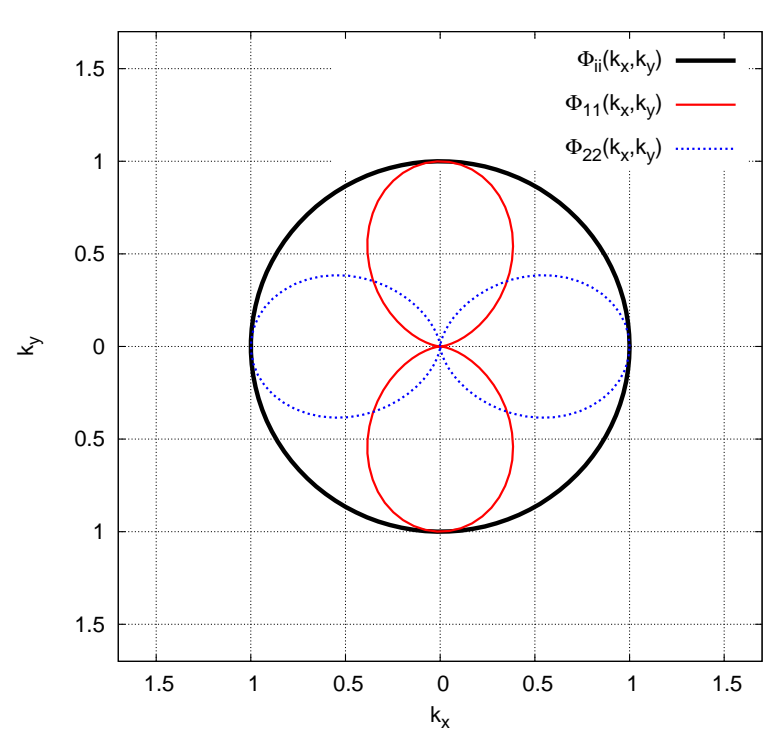

FIG. 4: (Color online) The variance of individual components of a divergence-free isotropic vector field is not isotropically distributed. Isovalues of two components $\Phi_{11}(\boldsymbol{k})$ and $\Phi_{22}(\boldsymbol{k})$ are plotted as well as their isotropic sum.

cal vortex tubes in rotating turbulence, as illustrated in figure 1. The distribution of energy in wavelet space, i.e. the wavelet coefficients in the different boxes, allows a direct quantification of anisotropy. The directional energy, $e_{(d)}$ is obtained by summing the energy over all boxes in wavelet space in a particular direction (illustrated in Figure 2 , on the left, for a horizontal direction). It can be stressed here that the vertical direction in wavelet space contains a particular discrete dyadic cone in Fourier space around the $k_{z}$-axis, similar to the cones in $[6,20]$. We can therefore anticipate that the energy in this direction will be reduced in the rotating case and enhanced in the stratified case.

In figure 5 the directional energy is shown for the three different velocity components $u, v$ and $w$ as a percentage with respect to the total energy of $\boldsymbol{u}$. In the present work we focus on the three principal directions $x, y$ and $z$. The four diagonal directions, which contain the remaining part of the energy, are not shown. For the divergence-free Gaussian noise, if one considers one velocity component, the directional distribution is not isotropic. Longitudinal energy (e.g. $u$ in the $x$-direction) is smaller than the transversal components (e.g. $u$ in the $y$-direction). The reason for this is the incompressibility constraint, as illustrated in figure 4: the Riesz projector reduces the energy in the longitudinal direction in favour of the transversal ones. The directional energy of isotropic turbulence behaves very similar to the Gaussian field: the longitudinal energy is smaller than the transversal energy. For rotating turbulence we see a similar picture, even though the directional energy is reduced in the $z$-direction. Indeed the formation of columnar structures reduces the spatial variation in this direction. The reduction of the energy in the $z$-direction is however moderate and twodimensionalization is not achieved. The picture is dramatically different for stratified turbulence in which all components are reduced in favour of the $u$ and $v$ energy in the $z$-direction. This energy distribution clearly corresponds to a vertically sheared horizontal flow. The flow is close to the two-component limit.

\section{B. Directional flatness}

The standard deviation of the spatial distribution of the scale-dependent energy involves the square root of fourth-order moments of the wavelet coefficients. It can be directly related to the flatness. For a component $u$ of the velocity field we introduce the directional scaledependent flatness:

$$
F_{(j, d)}^{u}=\frac{\left\langle\tilde{u}_{\lambda}^{4}\right\rangle_{(j, d)}}{\left\langle\tilde{u}_{\lambda}^{2}\right\rangle_{(j, d)}^{2}}
$$

in which averaging is performed only over the position $\boldsymbol{i}$. This can be related to the standard deviation of the spectral distribution of $u$ by:

$$
F_{(j, d)}^{u}=\left(\frac{\sigma_{\tilde{E}_{u}}\left(k_{j}, d\right)}{\tilde{E}_{u}\left(k_{j}, d\right)}\right)^{2}+1
$$

in which the spectrum $\tilde{E}_{u}\left(k_{j}, d\right)$ and its standard deviation $\sigma_{\tilde{E}_{u}}\left(k_{j}, d\right)$ are defined as in (4) and (5) by using only one component of the velocity in one direction $d$ of wavelet space. The scale-dependent directional flatness is thus a measure for the relative spatial fluctuation of the directional spectral energy density. It should therefore be an adequate measure for intermittency in the sense of Kraichnan [9]: extreme relative intensity fluctuations at the small scales should translate into large deviations of $F_{(j, d)}^{u}$ from its Gaussian value 3. Furthermore, the physical space locality of the wavelets should allow to investigate inertial range intermittency if the Reynolds number is sufficiently high.

In figure 6 the directional flatness is plotted versus wavenumber for the different flow fields. For the divergence-free Gaussian white noise, the flatness is as expected equal to three. For all the other flows the intermediate scales are close to this value. For isotropic turbulence the small scale flatness increases. This departure from Gaussianity characterizes an increased intermittency in the small scales. The longitudinal flatness is smaller than the transversal flatness.

An explanation for the increase of intermittency as a function of scale can be given starting with the theory of stochastic distortion of turbulence, proposed by Nazarenko et al. [15] for the dynamo problem and Dubrulle et al. [16] for Navier-Stokes turbulence. According to their model, small-scale wavepackets (in our case wavelets), initially isotropically distributed, get deformed by small-wavenumber induced strain. This results in cactus-leaf shaped distributions with one small, 

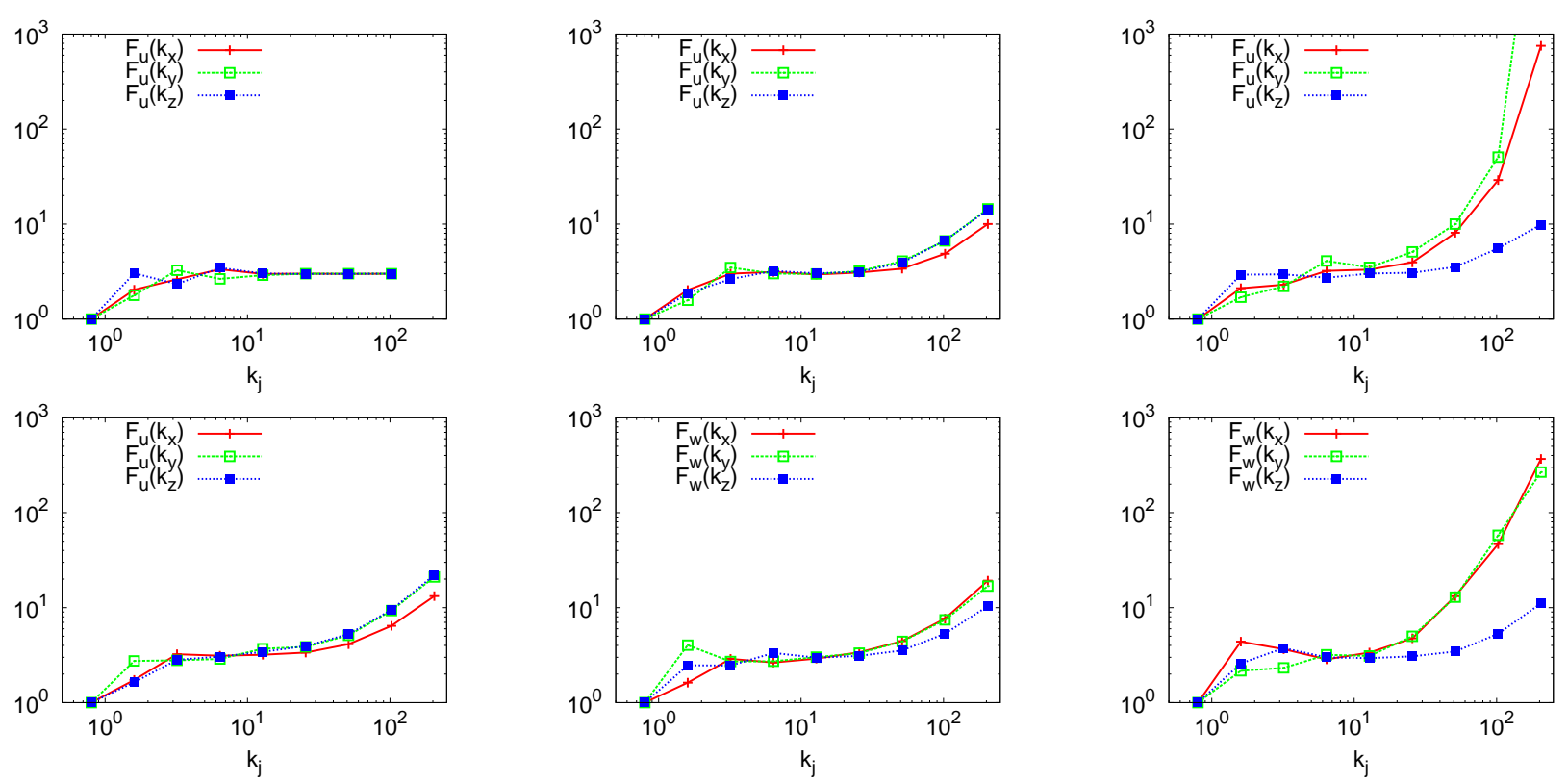

FIG. 6: (Color online) Directional scale-dependent flatness for Gaussian white noise (left top) and isotropic turbulence $u$ component (left bottom), rotating turbulence, $u$ and $w$ components (middle) and stratified turbulence, $u$ and $w$ components (right).

[3] V.A. Sandborn. J. Fluid Mech, 6, 221, (1938).

[4] G.K. Batchelor and A.A. Townsend. Proc. Roy. Soc. Lond. A, 199, 238, (1949).

[5] J.J. Riley, R.W. Metcalfe, and M.A. Weissman. In Proc. AIP conference, Nonlinear properties of internal waves, page 79. AIP, (1981).

[6] F.S. Godeferd and C. Cambon. Phys. Fluids, 6, 2084, (1994).

[7] C. Cambon. Eur. J. Mech. B, 20, 489, (2001).

[8] C. Brun and A. Pumir. Phys. Rev. E, 63, 056313, (2001).

[9] R. H. Kraichnan. Phys. Fluids, 10, 2080, (1967).

[10] I. Arad, V.S. L'vov, and I. Procaccia. Phys. Rev. E, 59, 6753, (1999).

[11] A. Babiano, C. Basdevant, and R. Sadourny. J. Atmos. Sci., 42, 941, (1985).

[12] K. Schneider, M. Farge, and N. Kevlahan. In Woods Hole Mathematics : Perspectives in Mathematics and Physics, pages 302-328. World Scientific, (2004).

[13] C. Meneveau. J. Fluid Mech., 232, 469, (1991).

[14] M. Farge, Y. Guezennec, C.M. Ho, and C. Meneveau. In
CTR Summer Report 1990, pages 331-343, (1990).

[15] S. Nazarenko, R.J. West, and O. Zaboronski. Phys. Rev. E, 68, 026311, (2003).

[16] B. Dubrulle, J.-P. Laval, S. Nazarenko, and O. Zaboronski. J. Fluid Mech., 520, 1, (2004).

[17] L. Liechtenstein, F.S. Godeferd, and C. Cambon. J. Turbul., 6, 1, (2005).

[18] M. Farge. Annu. Rev. Fluid Mech., 24, 395, (1992).

[19] S. Mallat. A wavelet tour of signal processing. Academic Press, (1998).

[20] C. Cambon and L. Jacquin. J. Fluid Mech., 202, 295, (1989).

[21] Y. Li and C. Meneveau. Phys. Rev. Lett., 95, 164502, (2005).

[22] M. Chertkov, A. Pumir and B. I. Shraiman Phys. Fluids, 11, 2394, (1999).

[23] L. Chevillard and C. Meneveau. Phys. Rev. Lett., 97, 174501, (2006). 